\title{
Block-Based Performance Measures for MIMO OFDM Beamforming Systems
}

\author{
Krishna P. Kongara, Ping-Heng Kuo, Peter J. Smith, Member, IEEE, Lee M. Garth, and Alan Clark
}

\begin{abstract}
In this paper, we consider an adaptive modulation system with multiple-input-multiple-output (MIMO) antennas in conjunction with orthogonal frequency-division multiplexing (OFDM) operating over frequency-selective Rayleigh fading environments. In particular, we consider a type of beamforming with a maximum ratio transmission/maximum ratio combining (MRT-MRC) transceiver structure. For this system, we derive a central limit theorem for various block-based performance metrics. This motivates an accurate Gaussian approximation to the system data rate and the number of outages per OFDM block. In addition to the data rate and outage distributions, we also consider the subcarrier signal-to-noise ratio (SNR) as a process in the frequency domain and compute level crossing rates (LCRs) and average fade bandwidths (AFBs). Hence, we provide fundamental but novel results for the MIMO OFDM channel. The accuracy of these results is verified by Monte Carlo simulations, and applications to performance analysis and system design are discussed.
\end{abstract}

Index Terms-Adaptive modulation, average fade bandwidth (AFB), beamforming, eigenvalues, level crossing rate (LCR), multiple-input-multiple-output orthogonal frequencydivision multiplexing (MIMO OFDM), Rayleigh fading channels.

\section{INTRODUCTION}

$\mathbf{M}$ ULTIPLE-input-multiple-output orthogonal frequencydivision multiplexing (MIMO OFDM) systems, involving clever processing in the spatial and frequency domains, have been proposed for WiFi, WiMax, and fourth-generation cellular systems, as well as the IEEE 802.16 standard for wireless Internet access. In conjunction with this, adaptive modulation for MIMO OFDM systems has recently been a subject of intense research for next-generation mobile wireless systems [1]-[3]. This paper focuses on the statistical analysis of adaptive MIMO OFDM beamforming systems, providing important insight into the system throughput and the outage probability. In particular, we derive closed-form approximations for the

Manuscript received December 5, 2007; revised June 10, 2008. First published September 3, 2008; current version published May 11, 2009. The review of this paper was coordinated by Prof. W. Su.

K. P. Kongara and P. J. Smith are with the Department of Electrical and Computer Engineering, University of Canterbury, Christchurch 8140, New Zealand (e-mail: kpk15@student.canterbury.ac.nz; p.smith@elec.canterbury.ac.nz).

P.-H. Kuo is with the Department of Electrical and Computer Engineering, University of Canterbury, Christchurch 8140, New Zealand.

L. M. Garth is with the Department of Electrical and Computer Engineering, University of Canterbury, Christchurch 8140, New Zealand, and also with the Advanced Systems and Technologies Division, BAE Systems, Merrimack, NH, 03054 USA.

A. Clark is with the Networks Research Centre, British Telecommunications PLC, IP5 3RE Ipswich, U.K.

Color versions of one or more of the figures in this paper are available online at http://ieeexplore.iee.org.

Digital Object Identifier 10.1109/TVT.2008.2005102 cumulative distribution function (cdf) and the exact mean and variance of the number of bits transmitted per OFDM block and the number of outages per OFDM block. An OFDM block is defined as $N$ baseband-modulated symbols appended by a cyclic prefix. In addition, we analyze the level crossing rate (LCR) and the average fade bandwidth (AFB) of the subcarrier SNR across frequency. Results for the LCR and the AFB in the time domain are common. In this paper, the novelty is in the analysis of the LCR and the AFB for MIMO eigenmodes in the frequency domain.

Multiple antennas at the transmitter and the receiver create a MIMO wireless channel and can be used to provide spatial diversity to combat fluctuations in signal strength or fading in wireless channels [1]-[4]. MIMO wireless systems have been shown to provide a diversity gain that is equal to the product of the number of transmit antennas and the number of receive antennas in narrow-band channels. For the case of the complete knowledge of the channel state information (CSI) at the transmitter, full diversity can be achieved using transmit beamforming and receive combining [5]-[7]. Compared with space-time block codes, beamforming and combining provide an additional array gain and a low-complexity and flexible receiver structure. The CSI at the transmitter may be obtained from the receiver using a feedback control channel. The particular case of beamforming and combining using a feedback channel to convey the CSI has been investigated in [8] and [9].

It is well known that OFDM can be used to convert a broadband MIMO channel into multiple narrow-band MIMO channels: one for each tone or subcarrier [9], [10]. Then, beamforming and combining can be independently applied to each subcarrier. We presume the availability of some minimum feedback link from the receiver to the transmitter. Furthermore, we assume that each transmit antenna is allocated a fixed amount of power and that no power reallocation is performed across the transmit antennas.

The statistical variation of various metrics across subcarriers in OFDM is a fundamental problem in OFDM performance, but one that has received little attention probably due to the mathematical challenges that are involved. Consider the complexity of a complete description of the statistics of an OFDM block. Even in the single-input-single-output (SISO) case, this would require the joint distribution of $N$ correlated Rayleigh variables, where $N$ represents the number of subcarriers in the OFDM block. The probability density function (pdf) of a single Rayleigh variable is trivial, the bivariate density requires a Bessel function [11], and the trivariate Rayleigh density can be written as an infinite sum of products of Bessel functions [12]. Clearly, the general form for $N$ subcarriers is completely 
infeasible. The corresponding results for MIMO OFDM are even more complex. In summary, a complete description of the statistics of an OFDM block is out of reach; however, there are still useful results that can be derived to yield some insight into the behavior of various performance metrics across frequency.

In this paper, we concentrate on the data rate for an OFDM block and the number of outages per block. The mean results for such metrics can be attained from a single subcarrier; however, these results do not yield information about the likelihood of a block with many outages or low data rates. Such distributional information requires more complete analysis across frequency. This characterization of the system across frequency is the focus of this paper. In addition to considering the data rate and outage distributions, we also consider the subcarrier SNR as a process in the frequency domain and compute LCRs and AFBs. Hence, we provide fundamental but novel results for the MIMO OFDM channel. Furthermore, our analysis can also be used as a framework for other studies where the behavior over frequency is important. For example, similar approaches in [13] and [14] have led to results on the capacity of MIMO OFDM systems and the bit error rate (BER) of SISO OFDM systems.

Applications of this paper can be found in performance analysis and design. The data rate and outage distributions give more complete performance results than previously available. In particular, we are able to investigate the variation in the data rate and outage numbers and assess the probabilities of low data rates and high numbers of outages. The LCR and AFB results give a useful design tool that can be used in the selection of subbands, space-time-frequency coding schemes, pilot selection, and other processes that depend on the channel behavior across frequency.

Based on the new analytic joint cdf and joint complementary cdf results, we present new approximations to the number of bits transmitted and the number of outages per OFDM block. In particular, we present a new closed-form Gaussian approximation, which is shown to be extremely accurate for many different systems and channel scenarios. These novel Gaussian approximations can be used as a benchmark for MIMO OFDM maximum ratio transmission/maximum ratio combining (MRTMRC) system performance under fading channels.

The rest of this paper is organized as follows. In Section II, we outline key assumptions and notations that are used in the description of MIMO OFDM systems, and we present some fundamental background and mathematical formulations for MIMO channels and adaptive modulation system analysis. The MIMO OFDM system analysis and the derivation of the relevant cdf's are presented in Section III. Our LCR and AFB analysis is presented in Section IV. We verify our analysis using Monte Carlo simulations in Section V. Finally, we conclude this paper in Section VI.

\section{MIMO OFDM SYSTEM}

We assume a familiarity with OFDM and MIMO OFDM systems [15]-[17]. We consider an adaptive MIMO OFDM beamforming system transmitting over $N$ subcarriers with $N_{T}$ antennas at the transmitter and $N_{R}$ antennas at the receiver. The system transmits data symbol $S_{t, k}$ on the $k$ th subcarrier during the $t$ th discrete time interval, for $t \in \mathbb{Z}$ and $k \in\{1,2, \ldots, N\}$, where $S_{t, k} \in \mathbb{R}^{2}$ is from some 2-D symbol constellation. We refer to the superposition of all $N$ modulated subcarriers during the $t$ th time interval as the $t$ th OFDM block. We assume that each subcarrier occupies a subchannel of bandwidth $\Delta f$ (in hertz), such that the total bandwidth is $B=N \Delta f$, with block duration $T=1 / \Delta f$. We denote the center frequency of each subchannel as $f_{k}$ so that $f_{k+1}-f_{k}=\Delta f$. Furthermore, each subcarrier symbol is transmitted with equal energy $E_{s}$ such that the total average transmitted energy is $E_{N}=N E_{s}$. The analysis that is developed in this paper is across frequency, and we do not investigate the variation across time. Hence, we consider an arbitrary time interval, and let $S_{t, k}$ be replaced by $S_{k}$, which is the symbol on the $k$ th subcarrier. Similarly, other variables such as beamforming vectors and channel matrices will be denoted as functions of frequency $k$ but not of time.

At the transmitter, the $k$ th subcarrier modulates the symbol $S_{k}$ using the beamforming vector (or weight vector) $\boldsymbol{b}_{k}$. We assume that the sampled impulse response of the channel is shorter than the cyclic prefix. After removing the cyclic prefix, the channel for the $k$ th subcarrier after the discrete Fourier transform can then be described as an $N_{R} \times N_{T}$ complex channel matrix $\boldsymbol{H}_{k}$. Considering a beamforming-combining system, the output of the combiner at the receiver on the $k$ th subcarrier can be written as

$$
R_{k}=\boldsymbol{z}_{k}^{\dagger} \boldsymbol{H}_{k} \boldsymbol{b}_{k} S_{k}+\boldsymbol{z}_{k}^{\dagger} \boldsymbol{n}_{k}
$$

where $\dagger$ represents the conjugate transpose, $\boldsymbol{z}_{k}$ is the combiner weight vector, $\boldsymbol{H}_{k}$ is the narrow-band channel transfer function for subcarrier $k, b_{k}$ is the beamforming vector, and $S_{k}$ is the transmitted symbol. The noise vector is denoted by $\boldsymbol{n}_{k}$ with independent identically distributed (i.i.d.) Gaussian entries that are distributed according to $\mathcal{C N}\left(0, \sigma^{2}\right)$. We also assume that the power is equally allocated across all of the subcarriers, so that $E\left[\left|S_{k}\right|^{2}\right]=E_{s}$ is a constant, and set $\left\|\boldsymbol{b}_{k}\right\|=1$ to reflect the power constraint at the transmitter, where $\|\cdot\|$ denotes the Euclidean norm. As in [18] and [19], appropriate power allocation could be considered. In this paper, we focus on the case where equal power is allocated among all the subcarriers.

Given that the signal model in (1) is identical to that of a narrow-band system, $\boldsymbol{b}_{k}$ and $\boldsymbol{z}_{k}$ can be chosen to independently maximize the SNR for each subcarrier using the principles of beamforming and MRC [5], [8] for narrow-band MIMO systems. In the MIMO OFDM system under consideration, for a given beamforming vector $\boldsymbol{b}_{k}$, the combining vector $\boldsymbol{z}_{k}$ that maximizes the SNR is given by [8]

$$
\boldsymbol{z}_{k}=\frac{\boldsymbol{H}_{k} \boldsymbol{b}_{k}}{\left\|\boldsymbol{H}_{k} \boldsymbol{b}_{k}\right\|} .
$$

The maximum SNR that can be achieved is given by $E_{s} / \sigma^{2}\left\|\boldsymbol{H}_{k} \boldsymbol{b}_{k}\right\|^{2}$, as shown in [5] and [8].

The overall maximum SNR is achieved if the beamforming weight vector is proportional to the eigenvector corresponding to the maximum eigenvalue $\lambda_{\max }^{(k)}$ of $\boldsymbol{H}_{k} \boldsymbol{H}_{k}^{\dagger}$. This transmission scheme is commonly described as MRT-MRC, which achieves full diversity and the full array gain in Rayleigh fading 
channels. Substituting this eigenvector into (2), the resulting optimal SNR can be written as

$$
\gamma_{\max }^{(k)}=\frac{E_{s}}{\sigma^{2}} \lambda_{\max }^{(k)}
$$

where $E_{s} / \sigma^{2}$ denotes the average SNR per branch, and (1) can be replaced by

$$
R_{k}=\sqrt{\lambda_{\max }^{(k)}} S_{k}+\tilde{n}
$$

where $\widetilde{n} \sim \mathcal{C N}\left(0, \sigma^{2}\right)$ is a complex Gaussian noise term that is independent of $\lambda_{\max }^{(k)}$. This simple formulation of the received signal is a necessary result for the forthcoming modulation switching threshold calculation. From (3), we see that the subcarrier SNR $\gamma_{\max }^{(k)}$ is proportional to $\lambda_{\max }^{(k)}$. Hence, the adaptive system can select the modulation scheme based on the maximum eigenvalue.

\section{A. Frequency-Selective SISO Channel}

Again, we assume a familiarity with frequency-selective Rayleigh fading channels and use the well-known Jakes' model [20]. We make the general assumption of a frequency-selective Rayleigh fading channel that is wide-sense stationary with uncorrelated isotropic scattering. In addition, we consider that the channel gain process in the time domain is static within a single OFDM block, but may change from block to block. Furthermore, we presume that the delay autocorrelation function may be described as an exponential delay power profile with rms delay $\tau_{d}$. However, note that the analysis developed later does not depend on the type of delay power profile. An exponential profile is chosen here, as it is a commonly used model. As before, we select an arbitrary time point and only consider the variation across frequency.

Then, we may write the $k_{1}$ th and $k_{2}$ th subchannel gains as

$$
\begin{aligned}
& H_{k_{1}}=X_{k_{1}}+j Y_{k_{1}} \\
& H_{k_{2}}=X_{k_{2}}+j Y_{k_{2}}
\end{aligned}
$$

where $X_{k_{1}}, Y_{k_{1}}, X_{k_{2}}$, and $Y_{k_{2}}$ are identically distributed zeromean Gaussian random variables. Without loss of generality, we may set $E\left[X_{k}^{2}\right]=E\left[Y_{k}^{2}\right]=1 / 2$, for all $k$. Following [20], we may then write the cross correlations

$$
\begin{aligned}
& E\left[X_{k_{1}} X_{k_{2}}\right]=E\left[Y_{k_{1}} Y_{k_{2}}\right]=\frac{1}{2} \frac{1}{1+\left(2 \pi \tau_{d} \Delta f \Delta k\right)^{2}} \\
& E\left[X_{k_{1}} Y_{k_{1}}\right]=E\left[X_{k_{2}} Y_{k_{2}}\right]=0 \\
& E\left[X_{k_{1}} Y_{k_{2}}\right]=-E\left[X_{k_{2}} Y_{k_{1}}\right]=-\left(2 \pi \Delta f \Delta k \tau_{d}\right) E\left[X_{k_{1}} X_{k_{2}}\right]
\end{aligned}
$$

where $\Delta k=\left|k_{1}-k_{2}\right|$. With these definitions, we obtain the correlation function

$$
\rho_{f}(\Delta k \Delta f)=E\left[H_{k 1} H_{k 2}^{*}\right]=\frac{1+j 2 \pi \tau_{d} \Delta f \Delta k}{1+\left(2 \pi \tau_{d} \Delta f \Delta k\right)^{2}} .
$$

Note that from (5), the marginal distribution of each channel gain $\left|H_{k}\right|^{2}$ follows an exponential distribution with $E\left[\left|H_{k}\right|^{2}\right]=$ $1, \operatorname{var}\left|H_{k}\right|^{2}=1$, and

$$
\operatorname{corr}\left(\left|H_{k}\right|^{2},\left|H_{k+\Delta k}\right|^{2}\right)=\frac{1}{1+\left(2 \pi \Delta f \Delta k \tau_{d}\right)^{2}}
$$

where $\operatorname{corr}(\cdot, \cdot)$ represents the correlation coefficient.

\section{B. Frequency-Selective MIMO Channel}

We assume independent channel coefficients in the $N_{R} \times N_{T}$ channel matrix $\boldsymbol{H}_{k}$ for all subcarriers $k$. This is a reasonable assumption in urban environments or when the antenna spacings and the angle spreads at the transmitter and the receiver are large. Across frequency, the statistics of each entry of $\boldsymbol{H}_{k}$ are governed by Jakes' model described in Section II-A. Hence, we consider correlations in frequency, but assume spatial independence. To the best of our knowledge, the equivalent derivation of the required joint cdf of the maximum eigenvalue in bin $i$ and bin $(i+k)$ is very difficult in the presence of spatial correlation. The nonzero eigenvalues of $\boldsymbol{H}_{k} \boldsymbol{H}_{k}^{\dagger}$ are denoted by $\lambda_{1}^{(k)}>\lambda_{2}^{(k)}>\cdots>\lambda_{m}^{(k)}$, where $m=\min \left(N_{R}, N_{T}\right)$, and the maximum eigenvalue is denoted by $\lambda_{\max }^{(k)}=\lambda_{1}^{(k)}$. The cdf of $\lambda_{\max }^{(k)}$ is known [5]-[8], [21] and is denoted by $F(x)=\operatorname{Prob}\left(\lambda_{\max }^{(k)} \leq x\right)$. For a generic subcarrier, we omit the superscript and write $\lambda_{\max }$. We will also require the notation $n=\max \left(N_{R}, N_{T}\right)$ in later sections.

\section{Adaptive Modulation System}

Adaptive modulation is a technique that increases the spectral efficiency under changing channel conditions. In more favorable channel conditions, a higher number of bits per symbol can be transmitted, whereas in less-favorable conditions, modulation is downgraded to a less-spectrally efficient constellation. It is generally assumed that, in adaptive modulation, the system attempts to maintain a constant target BER while maximizing the spectral efficiency [22]. The CSI is fed back from the receiver to the transmitter, and the maximum eigenvalue for each subcarrier is compared with a set of fixed thresholds $\left\{T_{1}, T_{2}, \ldots, T_{L+1}\right\}$, where $L$ is the number of alternative modulation modes. In this paper, the feedback channel is assumed to be ideal (error free). If the maximum eigenvalue lies between thresholds $T_{i}$ and $T_{i+1}$, then the $i$ th modulation mode is used by the transmitter. The thresholds are calculated by combining the available information regarding the channel fading model, the target BER, and the spectral efficiency of various possible modulation modes.

Here, we use an adaptive modulation scheme in which the estimated subcarrier SNR values (via the maximum eigenvalues) are used to adjust the modulation scheme. We ignore any guard interval or cyclic prefix in the OFDM block. Furthermore, we consider seven modulation options: outage, binary phase-shift keying, quaternary phase-shift keying, eight-phase-shift keying (8-PSK), 16-quadratic-amplitude modulation (16-QAM), 32-QAM, and 64-QAM. Note that it is quite common to switch 
TABLE I

MAXimum EigenValue Thresholds For Two TARget BER VAlues

\begin{tabular}{|l|l|l|}
\hline Modulations & \multicolumn{2}{|c|}{ Maximum eigenvalue thresholds } \\
\hline \hline & Target $\mathrm{BER}=10^{-3}$ & Target $\mathrm{BER}=10^{-2}$ \\
\hline Outage & $0 \leq \lambda_{\max }<0.331$ & $0 \leq \lambda_{\max }<0.194$ \\
BPSK & $0.331 \leq \lambda_{\max }<1.270$ & $0.194 \leq \lambda_{\max }<0.745$ \\
QPSK & $1.270 \leq \lambda_{\max }<4.873$ & $0.745 \leq \lambda_{\max }<2.861$ \\
8-PSK & $4.873 \leq \lambda_{\max }<6.622$ & $2.861 \leq \lambda_{\max }<3.771$ \\
16-QAM & $6.622 \leq \lambda_{\max }<13.785$ & $3.771 \leq \lambda_{\max }<7.794$ \\
32-QAM & $13.785 \leq \lambda_{\max }<28.014$ & $7.794 \leq \lambda_{\max }<15.840$ \\
64-QAM & $28.014 \leq \lambda_{\max }$ & $15.840 \leq \lambda_{\max }$ \\
\hline
\end{tabular}

off the weakest subcarriers [23], [24]. The SNR boundaries for switching between the modulation schemes are obtained using the approximate method for $M$-PSK and square $M$-QAM presented in [25], which are valid for received signals of the form given in (4). For a target BER of $p_{e}$, these approximations are given by

$$
\begin{aligned}
\mathrm{SNR}_{\mathrm{MPSK}} & \approx-\frac{1}{8} \ln \left(4 p_{e}\right) 2^{1.94 \frac{\ln (M)}{\ln (2)}} \\
\mathrm{SNR}_{\mathrm{MQAM}} & \approx-\frac{\overline{3}}{3}(M-1) \ln \left(5 p_{e}\right) .
\end{aligned}
$$

Using (3), we can obtain the modulation switching thresholds from the following expression:

$$
\frac{E_{s}}{\sigma^{2}} \lambda_{\max }=\mathrm{SNR}_{\gamma}
$$

where $\gamma=$ MQAM or MPSK. Substituting (9) or (10) into (11) gives threshold values for $\lambda_{\max }$, which can be used to implement the adaptive modulation scheme. The threshold values are summarized in Table I, where we have assumed an average SNR per branch equal to $9 \mathrm{~dB}$ and target BER values of $10^{-2}$ and $10^{-3}$. Note that exact BER results are also available; however, the improved accuracy in threshold calculation has a marginal effect on system performance, and the exact results are more cumbersome.

\section{Performance Metrics}

Most existing work on OFDM focuses on the mean performance and relies on the results for a single subcarrier, which are usually straightforward. For example, the mean symbol error rate (SER) of our system is simply SER = $\operatorname{Prob}\left(R_{k}\right.$ is not decoded as $\left.S_{k}\right)$, and the outage of each subcarrier is $\operatorname{Prob}\left(\lambda_{\max }^{(k)}<T\right)$, where $T$ is some threshold below which the channel is deemed to be in outage [7], [26]. These results are identical for every subcarrier, and such metrics give mean results with no indication as to the behavior of the whole block. In this paper, we consider block-based metrics such as the number of outages in the block and the data rate of the block. Extensions to other metrics such as BER and capacity [13] may also be possible.

Consider the binary-valued function

$$
B_{k}= \begin{cases}0, & \text { if } 0 \leq \lambda_{\max }^{(k)}<T \\ 1, & \text { if } T \leq \lambda_{\max }^{(k)}<\infty\end{cases}
$$

where $T$ is the threshold value below which modulation is suspended, i.e., an outage occurs. The function $B_{k}$ simply counts whether the $k$ th bin is ON or OFF. Also, consider the more general function

$$
W_{k}= \begin{cases}w_{1}, & \text { if } T_{1} \leq \lambda_{\max }^{(k)}<T_{2} \\ w_{2}, & \text { if } T_{2} \leq \lambda_{\max }^{(k)}<T_{3} \\ \vdots & \\ w_{L}, & \text { if } T_{L} \leq \lambda_{\max }^{(k)}<T_{L+1}\end{cases}
$$

which includes any metric that measures a fixed criterion based on $\lambda_{\max }^{(k)}$ in each bin. If $w_{i}$ is the number of bits that are used in the $i$ th modulation scheme, then $W=\sum_{k=1}^{N} W_{k}$ counts the total number of bits that are transmitted per OFDM block, and $B=\sum_{k=1}^{N} B_{k}$ gives the total number of times the modulation is ON per OFDM block. Since the number of outages in the block is $N-B$, we note that $B$ gives outage information. Similarly, the data rate of the block is $W / T$; therefore, $W$ gives the data rate. Since $B$ is a special case of $W$, we consider only $W$ in the following analysis. The regions that define function $W_{k}$ in (13) are also denoted by $R_{i}=\left[T_{i}, T_{i+1}\right)$.

\section{MIMO OFDM SYSTEM ANALYSIS}

As discussed in Section I, the exact distribution of $W$ is prohibitively complex due to the difficulties in using the joint pdf of $\left(\lambda_{\max }^{(1)}, \lambda_{\max }^{(2)}, \ldots, \lambda_{\max }^{(N)}\right)$. Alternatively, since $W$ is a sum of random variables, for a large number of subcarriers, we might suppose that the distribution of $W$ is approximately Gaussian, based on some variation of the central limit theorem (CLT). However, (6) shows that the correlations $E\left[X_{k 1} Y_{k 2}\right]$ decay with order $1 / \Delta k$ as the separation in frequency increases. This is a strongly correlated scenario, and ordinary CLT arguments for correlated variables may not be valid [27]. Hence, we use a theorem due to Arcones [28], [29] that is previously adapted for use in the OFDM research in [14]. The work in [14] was for SISO OFDM systems, but it is straightforward to extend it to the MIMO case. In this paper, our contribution is in extending the CLT to MIMO-OFDM MRT-MRC systems and applying it to data rate and outage analysis rather than capacity.

We state the Arcones theorem below, which applies to the case where the number of subcarriers $N$ increases, and $\Delta f$ remains fixed. Hence, we have a CLT for the case of an increasing bandwidth. Note that, as the bandwidth increases, for fixed $\Delta f$ and $E_{s}$, the total power will also increase. Thus, the CLT assumes that the power indefinitely increases as the number of subcarriers increases. Although this is unrealistic, the main purpose of the CLT is to validate the use of a Gaussian approximation for the finite bandwidth case, and here, the problem of increasing power is not an issue.

Theorem 1 (Arcones-de Naranjo): Let $\left\{\boldsymbol{X}_{j}\right\}_{j=1}^{\infty}$ be a stationary mean-zero sequence of Gaussian vectors in $\mathbb{R}^{d}$. Set $\boldsymbol{X}_{j}=\left(X_{j, 1}, \ldots, X_{j, d}\right)$. Let $g$ be a function on $\mathbb{R}^{d}$ with Hermite rank $\varphi(g)$ such that $1 \leq \varphi(g)<\infty$. Define

$$
r^{(p, q)}(k)=E\left[X_{m, p} X_{m+k, q}\right]
$$


for $k \in \mathbb{Z}$, where $m$ is any number that is large enough that $m \geq 1$ and $m+k \geq 1$. Suppose that

$$
\sum_{k=-\infty}^{\infty}\left|r^{(p, q)}(k)\right|^{\varphi(g)}<\infty
$$

for all $1 \leq p \leq d$ and $1 \leq q \leq d$. Then, as $N \rightarrow \infty$

$$
\frac{1}{\sqrt{N}} \sum_{j=1}^{N}\left\{g\left(\boldsymbol{X}_{j}\right)-E\left[g\left(\boldsymbol{X}_{j}\right)\right]\right\} \stackrel{D}{\longrightarrow} \mathcal{N}\left(0, \sigma_{g}^{2}\right)
$$

where " $\stackrel{D}{\longrightarrow}$ " denotes the "convergence in distribution," and

$$
\begin{aligned}
& \sigma_{g}^{2}=E\left[\left(g\left(\boldsymbol{X}_{1}\right)-E g\left(\boldsymbol{X}_{1}\right)\right)^{2}\right] \\
& +2 \sum_{k=1}^{\infty} E\left[\left(g\left(\boldsymbol{X}_{1}\right)-E\left[g\left(\boldsymbol{X}_{1}\right)\right]\right)\left(g\left(\boldsymbol{X}_{1+k}\right)-E\left[g\left(\boldsymbol{X}_{1+k}\right)\right]\right)\right] .
\end{aligned}
$$

We apply this theorem to the case where $\boldsymbol{X}_{j}=\operatorname{vec}\left(\boldsymbol{H}_{j}\right)$, $d=2 N_{R} N_{T}, N$ is the number of subcarriers, and $g\left(\boldsymbol{X}_{j}\right)=$ $g\left(\operatorname{vec}\left(\boldsymbol{H}_{j}\right)\right)=W_{j}$. From (6), condition (15) is simple to verify as long as the Hermite rank of $g$ is at least 2 [14]. Hence, in the Appendix, we demonstrate that the Hermite rank is at least 2, and the theorem then supplies a CLT for $W$.

The convergence in distribution described in (16) clearly motivates the following approximation. For large finite $N$, the distribution of $W$ may be approximated by a Gaussian random variable with mean $E[W]=N E\left[W_{i}\right]$ and variance

$$
\operatorname{var}[W]=N \operatorname{var}\left[W_{i}\right]+2 \sum_{k=1}^{N-1}(N-k) \operatorname{cov}\left[W_{1}, W_{1+k}\right] .
$$

Note that, since $W$ is discrete and nonnegative, alternative approximations based on the binomial distribution and its generalizations are certainly possible. However, the Gaussian approximation is surprisingly accurate, and such extensions are not considered. Since we are using a CLT for $W$, the approximate distribution solely depends on $E[W]$ and $\operatorname{var}[W]$. However, we have mean $E[W]=N E\left[W_{i}\right]$, where

$$
E\left[W_{i}\right]=\sum_{k=1}^{L} w_{k}\left[F\left(T_{k+1}\right)-F\left(T_{k}\right)\right]
$$

and the variance is given by (18), where

$$
\begin{gathered}
\operatorname{var}\left[W_{i}\right]=\sum_{k=1}^{L} w_{k}^{2}\left[F\left(T_{k+1}\right)-F\left(T_{k}\right)\right]-E\left[W_{i}\right]^{2} \\
\operatorname{cov}\left[W_{1}, W_{1+k}\right] \\
=E\left[W_{1} W_{1+k}\right]-E\left[W_{1}\right]^{2} \\
=\sum_{i=1}^{L} \sum_{j=1}^{L} w_{i} w_{j} \operatorname{Prob}\left(T_{i} \leq \lambda_{\max }^{(1)}<T_{i+1}\right. \\
\left.\quad T_{j} \leq \lambda_{\max }^{(k+1)}<T_{j+1}\right) \\
-E\left[W_{1}\right]^{2} .
\end{gathered}
$$

These equations can be evaluated if we know the marginal and joint probabilities of the maximum eigenvalues in bins 1 and $k+1$.

First, we consider the marginal distribution of $\lambda_{\max }$, where, for convenience, we omit the superscript. $E\left[W_{i}\right]$ and $\operatorname{var}\left[W_{i}\right]$ can be computed from the cdf of $\lambda_{\max }$. This cdf is well known [5]-[8], [21] and can be computed in a determinant form [6], [8], [21] or by using the pdf given in [5] and [7]. From the form of the joint density for $\lambda_{1}^{(k)}, \lambda_{2}^{(k)}, \ldots, \lambda_{m}^{(k)}$ (see, e.g., [30]) and from [5] and [7], it is straightforward to see that the cdf is a linear combination of the form

$$
F(x)=\operatorname{Prob}\left(\lambda_{\max } \leq x\right)=\gamma_{0}+\sum_{r=1}^{m} \sum_{s=0}^{(m+n-2 r) r} \gamma_{r, s} x^{s} e^{-r x} .
$$

The coefficients $\gamma_{0}$ and $\gamma_{r, s}$ can be found using the algorithm in [7]; however, we simply compute them using a symbolic manipulation package such as Maple. Next, we consider the joint distribution of $\lambda_{\max }^{(1)}$ and $\lambda_{\max }^{(k+1)}$.

\section{A. Derivation of the Joint $C D F$}

To complete the calculation of (18)-(21), we require probabilities of the form

$$
\begin{aligned}
\operatorname{Prob} & \left(T_{i} \leq \lambda_{\max }^{(1)}<T_{i+1}, T_{j} \leq \lambda_{\max }^{(k+1)}<T_{j+1}\right) \\
& =F_{k}\left(T_{i+1}, T_{j+1}\right)-F\left(T_{i}\right)-F\left(T_{j}\right)+F_{k}\left(T_{i}, T_{j}\right)
\end{aligned}
$$

where $F_{k}(x, y)$ is the joint cdf of $\lambda_{\max }^{(1)}$ and $\lambda_{\max }^{(k+1)}$, which is defined by $F_{k}(x, y)=\operatorname{Prob}\left(\lambda_{\max }^{(1)} \leq x, \lambda_{\max }^{(k+1)} \leq y\right)$. Since the marginal cdf's $F\left(T_{i}\right)$ and $F\left(T_{j}\right)$ are already known from (22), we only require $F_{k}\left(T_{i}, T_{j}\right)$ to complete the derivation of $\operatorname{var}(W)$.

The calculation of the joint cdf of $\lambda_{\max }^{(1)}$ and $\lambda_{\max }^{(k+1)}$ relies on a result in [30], where the joint pdf of the ordered eigenvalues $\boldsymbol{\lambda}=\left(\lambda_{1}, \ldots, \lambda_{m}\right)=\left(\lambda_{1}^{(1)}, \ldots, \lambda_{m}^{(1)}\right)$ and $\boldsymbol{w}=\left(w_{1}, \ldots\right.$, $\left.w_{m}\right)=\left(\lambda_{1}^{(k+1)}, \ldots, \lambda_{m}^{(k+1)}\right)$ is shown to be

$$
\begin{aligned}
f_{o}(\boldsymbol{w}, \boldsymbol{\lambda})= & C_{o}\left(1-\rho^{2}\right)^{-m} \rho^{-m(n-1)} \\
& \times \exp \left\{-\frac{1}{1-\rho^{2}} \sum_{k=1}^{m}\left(w_{k}+\lambda_{k}\right)\right\} \\
& \times \prod_{i<j}^{m}\left[\left(\lambda_{i}-\lambda_{j}\right)\left(w_{i}-w_{j}\right)\right] \\
& \times\left|\left(\lambda_{i} w_{j}\right)^{(n-m) / 2} I_{n-m}\left(2 \sqrt{\mu \lambda_{i} w_{j}}\right)\right|
\end{aligned}
$$

where $\rho=\left|\rho_{f}(\Delta k \Delta f)\right|, \mu=\rho^{2}\left(1-\rho^{2}\right)^{-2}, \lambda_{1} \geq \lambda_{2} \geq \cdots \geq \lambda_{m}$, $w_{1} \geq w_{2} \geq \cdots \geq w_{m}$, and

$$
C_{o} \triangleq\left\{\prod_{k=1}^{m}[(n-k) !(m-k) !]\right\}^{-1} .
$$

In (24), the notation $\left|M_{i j}\right|$ refers to the determinant of an $m \times m$ matrix $M$ with the $(i, j)$ th element $M_{i j}$. Note the slight 
TABLE II

JoINT CDF VALUES COMPUTED USING (26)

\begin{tabular}{|lllllll|}
\hline 0.0000 & 0.0001 & 0.0007 & 0.0007 & 0.0007 & 0.0007 & 0.0007 \\
0.0001 & 0.0069 & 0.0578 & 0.0629 & 0.0643 & 0.0643 & 0.0643 \\
0.0007 & 0.0578 & 0.6673 & 0.7652 & 0.8031 & 0.8032 & 0.8032 \\
0.0007 & 0.0629 & 0.7652 & 0.8877 & 0.9389 & 0.9390 & 0.9390 \\
0.0007 & 0.0643 & 0.8031 & 0.9389 & 0.9996 & 0.9998 & 0.9998 \\
0.0007 & 0.0643 & 0.8032 & 0.9390 & 0.9998 & 1.0000 & 1.0000 \\
0.0007 & 0.0643 & 0.8032 & 0.9390 & 0.9998 & 1.0000 & 1.0000 \\
\hline
\end{tabular}

abuse of notation, where, for convenience, we have rewritten $\lambda_{i}^{(1)}$ and $\lambda_{i}^{(k+1)}$ as $\lambda_{i}$ and $w_{i}$, respectively. Using some determinant results in [31], we are able to integrate out $\left(\lambda_{2}, \ldots, \lambda_{m}\right)$ and $\left(w_{2}, \ldots, w_{m}\right)$ from $(24)$ to obtain the required cdf.

The details are given in the Appendix, and the final result can be written as

$$
F_{k}(x, y)=K\left|A_{i j}(x, y)\right|
$$

where $K=\left(1-\rho^{2}\right)^{-m} \rho^{-m(n-1)} C_{o}$, and $\left|A_{i j}(x, y)\right|$ represents the determinant of the $m \times m$ matrix $\mathbf{A}(x, y)$ with the $(i, j)$ th element given in (27), shown at the bottom of the page. In $(27), \gamma(\cdot, \cdot)$ is the incomplete gamma function $\gamma(\alpha, \beta)=$ $\int_{0}^{\beta} t^{\alpha-1} e^{-t} d t$, and $\delta$ is defined by $\delta=\left(1-\rho^{2}\right)^{-1}$.

Hence, the joint and marginal distributions can be found by computing $A_{i j}(x, y)$. Although the infinite series for $A_{i j}(x, y)$ is not desirable, we have found that the series converges quickly. For example, when we consider a $(4,4)$ MIMO OFDM system with $N=64$ and $\tau_{d}=100 \mathrm{~ns}$, the series in (27) converges in less than 45 iterations to within typical machine accuracy. For larger values of $\tau_{d}$ or smaller system sizes, the convergence is even faster. For these system parameters and a target BER of $10^{-3}$, the evaluation of $\operatorname{var}[W]$ requires the probabilities $F_{k}\left(T_{i}, T_{j}\right)$, for $i=1,2, \ldots, 7$ and $j=1,2, \ldots, 7$, for each subcarrier lag, $k=1,2, \ldots, N-1$. As a numerical example, the probabilities for $k=4$ are given in Table II, where the $(i, j)$ th entry is $F_{k}\left(T_{i}, T_{j}\right)$.

The marginal probabilities can then be obtained from Table II using $\operatorname{Prob}\left(T_{i} \leq \lambda_{\max }<T_{i+1}\right)=F_{k}\left(T_{i+1}, \infty\right)-$ $F_{k}\left(T_{i}, \infty\right)$, yielding $\left[\begin{array}{llllll}0.0007 & 0.0636 & 0.7389 & 0.1358 & 0.0608\end{array}\right.$ $0.00020 .0001]$. This provides an alternative method to computing the marginal distribution so that computing the coefficients in (22) is unnecessary. Utilizing the joint and marginal probabilities, the mean and the variance can be obtained readily. In Section $\mathrm{V}$, we compare our analytical results using this Gaussian fit method with the simulation data and then use them to study the effects of the various system parameters. Methods for the uncorrelated case such as [32] are available; however, extensions to correlated channels appear to be unknown.

\section{B. Results for the Worst Eigenchannel}

Although this paper focuses on transmission over the maximal eigenchannel, it is instructive to consider the worst eigenchannel as well. In systems where all of the eigenchannels are employed, such as MIMO singular value decomposition systems, this enables us to provide best- and worst-case analysis. For this scenario, the joint cdf of $\left(\lambda_{\min }^{(1)}, \lambda_{\min }^{(k+1)}\right)$ can be computed using a minor variation of the proof in the Appendix. Consider (55), which gives the joint probability $\operatorname{Prob}\left(\lambda_{\min }^{(1)} \geq\right.$ $\left.c, \lambda_{\max }^{(1)} \leq d, \lambda_{\min }^{(k+1)} \geq a, \lambda_{\max }^{(k+1)} \leq b\right)$. Instead of setting $a=0$ and $c=0$ to obtain a cdf for the maximum eigenvalues, we set $b=\infty$ and $d=\infty$ to obtain a complementary cdf for the minimum eigenvalues. This exactly gives the same result as in (27) except that the incomplete gamma function $\gamma(\alpha, \beta)$ in (27) is replaced by the "upper" incomplete gamma function $\Gamma(\alpha, \beta)=\Gamma(\alpha)-\gamma(\alpha, \beta)$. Also, the result is no longer the joint cdf but instead gives the complementary cdf $R_{k}(x, y)=$ $\operatorname{Prob}\left(\lambda_{\min }^{(1)}>x, \lambda_{\min }^{(k+1)}>y\right)$. In summary, we have

$$
R_{k}(x, y)=K\left|\widetilde{A}_{i j}(x, y)\right|
$$

where the entries $\widetilde{A}_{i j}(x, y)$ are defined in (29), shown at the bottom of the page.

The joint cdf of the minimum eigenvalues follows from

$$
\begin{aligned}
\operatorname{Prob}\left(\lambda_{\min }^{(1)}<x, \lambda_{\min }^{(k+1)}<y\right) & =1-R_{k}(x, \infty) \\
& -R_{k}(\infty, y)+R_{k}(x, y)
\end{aligned}
$$

Hence, we are also able to form a Gaussian approximation to $W$ in the case where the minimum eigenchannel is used.

\section{LCR AND AFB}

The scope is moved to another block-based perspective in this section. Here, we are interested in how the channel gain fluctuates over the bandwidth of the OFDM system. To be specific, we aim to derive analytical expressions for the LCR and the AFB in the frequency domain (written as $\mathrm{LCR}_{f}$ and

$$
A_{i j}(x, y)=\sum_{k=0}^{\infty} \frac{\mu^{k+\frac{n-m}{2}} \gamma(n-m+j+k, \delta y) \gamma(n-m+i+k, \delta x)}{\delta^{n m+j+k} \delta^{n-m+i+k} k !(k+n-m) !}
$$

$$
\widetilde{A}_{i j}(x, y)=\sum_{k=0}^{\infty} \frac{\mu^{k+n-m 2} \Gamma(n-m+j+k, \delta y) \Gamma(n-m+i+k, \delta x)}{\delta^{n-m+j+k} \delta^{n-m+i+k} k !(k+n-m) !}
$$


$\mathrm{AFB}_{f}$, respectively) for the subcarrier gains, including the link gain of SISO OFDM systems and the eigenmode gain of MIMO OFDM systems using MRT-MRC. The LCR is the rate at which a gain process $g(f)$ crosses a level $\mathrm{T}$ in either the positive or negative direction. Note that this is a rate for the gain process over frequency and not over time. Formally, the LCR can be defined as [20]

$$
\operatorname{LCR}_{f}(T)=\int_{0}^{\infty} \dot{g} f_{g, \dot{g}}(T, \dot{g}) d \dot{g}
$$

where $\dot{g}$ is the frequency derivative of $g$, and $f_{g, \dot{g}}(g, \dot{g})$ is the joint pdf of $g$ and $\dot{g}$. The AFB is simply derived from the LCR by

$$
\operatorname{AFB}_{f}(T)=\frac{\operatorname{Prob}(g<T)}{\operatorname{LCR}_{f}(T)} .
$$

All of our analysis is carried out under the assumption of an i.i.d. Rayleigh channel. The potential practical applications of our results are also discussed.

\section{A. Subcarrier Link Gain in SISO OFDM}

It is well known that the link gain of a SISO Rayleigh fading channel $|H|^{2}$ is a complex chi-squared process with one degree of freedom, as the gain is the sum of squares of two i.i.d. Gaussian components [the real and imaginary parts in (5)]. Hence, following [33], the corresponding LCR for the process in the frequency domain is given by

$$
\operatorname{LCR}_{f,|H|^{2}}(T)=\sqrt{\frac{-\ddot{\rho}_{f}(0) T}{\pi}} \exp (-T)
$$

where $T$ is the threshold level, and $\ddot{\rho}_{f}(0)$ is the second derivative of the correlation function of the underlying Gaussian process at $\Delta f=0$.

From (7), the correlation function of the underlying Gaussian process with frequency separation $\Delta f$ is given by

$$
\rho_{f}(\Delta f)=\frac{1+j 2 \pi \tau_{d} \Delta f}{1+\left(2 \pi \tau_{d} \Delta f\right)^{2}} .
$$

To determine $\ddot{\rho}_{f}(0)$, the correlation function is expanded into a polynomial in $\Delta f$ (valid for small $\Delta f$ ) as

$$
\rho_{f}(\Delta f) \approx 1+j 2 \pi \tau_{d} \Delta f-\frac{\left(2 \pi \tau_{d} \Delta f\right)^{2}}{2} .
$$

Hence, the curvature of $\rho_{f}(\Delta f)$ at $\Delta f=0$ can be trivially obtained by doubling the coefficient of the $(\Delta f)^{2}$ term in (35), yielding

$$
\ddot{\rho}_{f}(0)=-4 \pi^{2} \tau_{d}^{2} .
$$

A very simple closed-form LCR formula for $|H|^{2}$ in the frequency domain can, therefore, be obtained by substituting (36) into (33) and is given by

$$
\mathrm{LCR}_{f,|H|^{2}}(T)=2 \tau_{d} \sqrt{\pi T} \exp (-T) .
$$

Clearly, $\mathrm{LCR}_{f}$ is proportional to $\tau_{d}$, which agrees with the work in [34]. Additionally, the AFB can be computed using the wellknown relationship between the LCR and the AFB, i.e.,

$$
\operatorname{AFB}_{f,|H|^{2}}(T)=\frac{\operatorname{Prob}\left(|H|^{2}<T\right)}{\operatorname{LCR}_{f,|H|^{2}}(T)}=\frac{1-\exp (-T)}{2 \tau_{d} \sqrt{\pi T} \exp (-T)} .
$$

Formulas (37) and (38) are derived assuming that the subcarrier gain is a continuous process in frequency. The simulations, however, consider a discrete process over the $N$ frequencies $f_{1}, f_{2}, \ldots, f_{N}$. For small $\tau_{d}$ values such as $100 \mathrm{~ns}$ used in Figs. 4-6, this difference is not important, as the process is very smooth $\left(\left|\rho_{f}(\Delta f)\right| \approx 0.9813\right)$, and the continuous approximation is very accurate. Increasing the value of $\Delta f \tau_{d}$ results in a lower $\rho_{f}(\Delta f)$, and the process tends to become more discrete. This leads to reduced accuracy in formulas (37) and (38).

To ameliorate this problem, we can alternatively evaluate the LCR from the bivariate Rayleigh density [11], which is the joint density of a subcarrier envelope and its adjacent neighbor. Specifically, denoting $U=\left|H_{k}\right|$ and $Z=\left|H_{k+1}\right|$, the LCR can be computed as follows:

$$
\operatorname{LCR}_{f,|H|^{2}}=\frac{\operatorname{Prob}(U>T, Z<T)}{\Delta f} .
$$

Note that the joint distribution of $U$ and $Z$ is a bivariate Rayleigh, and the distribution function can be expressed as the infinite series [11]

$F_{U, Z}(u, z)=(1-\rho) \sum_{\ell=0}^{\infty} \rho^{\ell} \bar{\gamma}\left(\ell+1, \frac{u^{2}}{1-\rho}\right) \bar{\gamma}\left(\ell+1, \frac{z^{2}}{1-\rho}\right)$

where $\rho=\operatorname{corr}\left(\left|H_{k}\right|^{2},\left|H_{k+1}\right|^{2}\right)$ is given by (8), and $\bar{\gamma}(\alpha, \beta)=$ $\gamma(\alpha, \beta) / \Gamma(\alpha)$ is the standardized incomplete gamma function. Hence, the joint probability can be efficiently computed by employing (40).

\section{B. Subcarrier SNR in MIMO OFDM}

In this paper, we are interested in how the maximum eigenvalues and, hence, the subcarrier SNR values evolve with frequency in a MIMO OFDM channel. The analysis of the LCR and the average fade duration of MIMO eigenmodes over time has been investigated in [35] and [36]. In particular, a very simple method for LCR computation has been given in [36], and the application of this technique is extended here to derive the LCR and the AFB for MIMO eigenmodes in the frequency domain.

As shown in [35] and [36], the eigenvalues as well as the singular values $s=\sqrt{\lambda}$ can be accurately approximated by gamma processes. As a result, the LCR for the eigenvalue process can be approximated using

$\operatorname{LCR}_{f, \lambda}(T)=\frac{1}{2 \Gamma(r)} \sqrt{\frac{2\left|\ddot{R}_{s}(0)\right|}{\pi}}(\theta \sqrt{T})^{r-0.5} e^{(-\theta \sqrt{T})}$

where $r=\{E[s]\}^{2} / \operatorname{var} s$ and $\theta=E[s] / \operatorname{var} s$ are the shape and scale factors, respectively, of the gamma variable that 
approximates the singular value process. Note that these parameters solely depend on the first two moments of the singular value process and, hence, can be acquired from the distribution of the eigenvalues. More details on computing $E[s]$ and var $s$ can be found in [36]. Also, following the same argument as in [36], $\ddot{R}_{s}(0)$ is the curvature of the correlation function of the singular value $s$, which is given by

$$
\ddot{R}_{s}(0)=\frac{-\ddot{\rho}_{f}(0) \theta^{2}}{2 r} .
$$

Hence, from (36), it is trivial to see that

$$
\ddot{R}_{s}(0)=\frac{2 \pi^{2} \tau_{d}^{2} \theta^{2}}{r} .
$$

Substituting (43) into (41), we have the following closed-form LCR formula:

$$
\operatorname{LCR}_{f, \lambda}(T)=\sqrt{\frac{\pi}{r}} \frac{\tau_{d} \theta}{\Gamma(r)}(\theta \sqrt{T})^{r-0.5} e^{(-\theta \sqrt{T})} .
$$

From (44), we can conclude that the LCR for the eigenmode in the frequency domain is also proportional to $\tau_{d}$. This formula simply requires the first two moments (for $r$ and $\theta$ ) of the corresponding singular value process, both of which can be acquired from the Wishart distribution [37]. Moreover, the AFB for the eigenmode gain is easily computed using

$$
\operatorname{AFB}_{f, \lambda}(T)=\frac{\operatorname{Prob}(\lambda<T)}{\operatorname{LCR}_{f, \lambda}(T)}
$$

where $\operatorname{Prob}(\lambda<T)$ can be calculated using either its gamma approximation or the exact marginal density of the eigenvalue [37].

\section{Potential Practical Applications}

Equipped with knowledge regarding the fluctuations of the channel in the frequency domain, many of the major mechanisms and parameters of an adaptive OFDM system, such as the channel estimation method, the feedback overhead, and the power/bit allocation algorithms, can be designed in a more judicious manner. To be specific, in lieu of adjusting the transmission mode on a subcarrier-by-subcarrier basis (the feedback overhead of which is, intuitively, a heavy burden for the system) in adaptive OFDM schemes, many researchers have suggested aggregating consecutive subcarriers with similar gains into groups or clusters called "subbands" (see, e.g., [38] and [39]). Such a strategy can significantly decrease the resources that are required for the feedback and, thus, leads to a more efficient operation of the system despite the insignificant performance loss. Assuming that the adaptive system is switching between "transmission" and "outage," the LCR in the frequency domain and the AFB gauge the appropriate number of subcarriers that can be grouped in interpolation-based channel estimators, as well as the suitable size for the subbands. These parameter settings affect the overall performance and, hence, are important in the development of subcarrier grouping algorithms. For systems that switch among more than two transmission modes, the mode

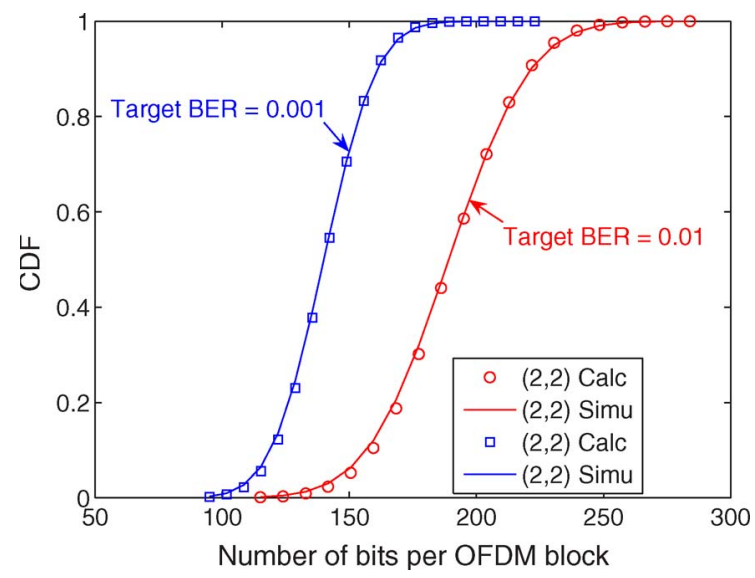

Fig. 1. CDF plots for the number of bits per OFDM block in a $(2,2)$ MIMO OFDM for two different target BERs $\left(N=64, \tau_{d}=100 \mathrm{~ns}\right)$.

entering rate (MER) and the average stay bandwidth (ASB) are of more interest. Further investigation of the MER and the ASB is, however, beyond the scope of this paper.

The LCR and the AFB are useful in many aspects of system design such as in selecting burst error correcting codes and the type of burst error correcting codes, and selecting the best length of a forward error correction block and the depth of interleaving to break up error bursts. Also, in adaptive OFDM systems, given the AFB curve, a subband of the subcarriers in the block can be chosen to use a single modulation format.

\section{Verification of ANALYSis With Simulation RESUlTS}

The simulations were carried out for a 64-subcarrier system and a 512-subcarrier system. Both systems have a subcarrier separation $\Delta f=0.3125 \mathrm{MHz}$, thus occupying bandwidths of 20 and $160 \mathrm{MHz}$, respectively. Also, a system carrier frequency of $5.725 \mathrm{GHz}$ (HyperLan 2 standard) was chosen. In our first set of results, we compare the Gaussian cdf based on the CLT using analytically derived mean and variance with subcarrier modulation statistics obtained from Monte Carlo simulations. We evaluate two systems with target BERs of $10^{-3}$ and $10^{-2}$, respectively. Furthermore, our simulations were carried out to observe the effect of the correlation across frequency on the approximating distributions. We consider mean delay spreads of 100 and $250 \mathrm{~ns}$, which give correlation coefficients of $\left|\rho_{f}(\Delta f)\right|=0.9813$ and 0.8977 , respectively, for an exponential power delay profile.

Figs. 1 and 2 show excellent agreement between the Gaussian approximation and the simulated data rates for OFDM blocks. Note that, in Fig. 1, we have only 64 subcarriers, and the correlation between adjacent subcarriers is of magnitude 0.9813 . Hence, the CLT is worked quite hard, but still yields excellent results. We observe that the Gaussian approximation is accurate for all target BERs, delay spreads, system sizes, and subcarrier numbers. We see that the distributions are tighter for nonsquare system sizes, lower target BERs, and larger delay spreads. Furthermore, we find a substantial variation in the data rate. With the adaptive scheme considered, the number of bits per block must lie in the region $[0,6 N]$ since a 6-bit symbol in 


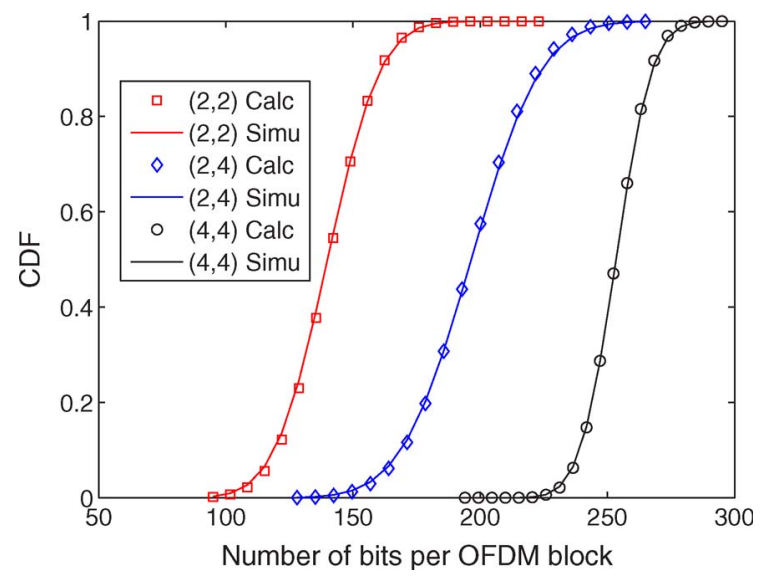

Fig. 2. CDF comparison between analysis and simulation for various MIMO system configurations $\left(N=64, \tau_{d}=100 \mathrm{~ns}\right.$, target BER $\left.=10^{-3}\right)$.

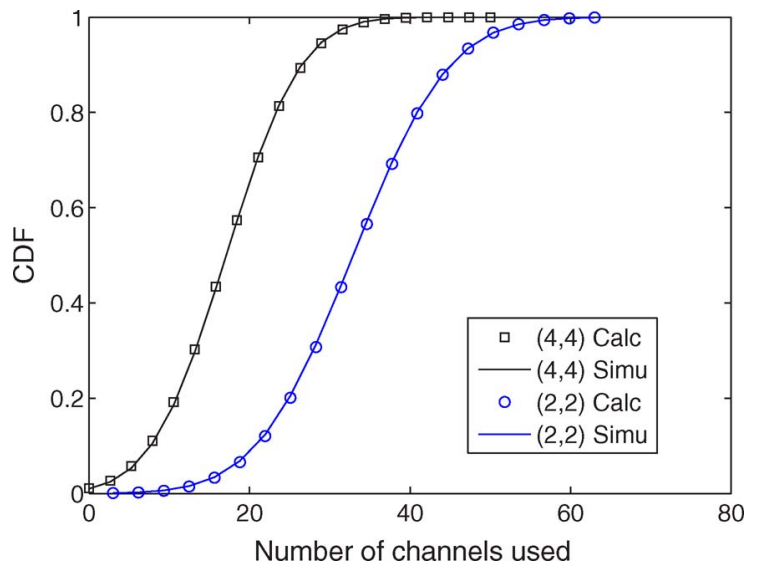

Fig. 3. CDF plots with transmission on the channel with minimum eigenmode $\lambda_{\min }\left(N=64, \tau_{d}=100 \mathrm{~ns}\right.$, target BER $\left.=10^{-3}\right)$.

64-QAM is the maximum. Considering Fig. 1, we see that for a target BER of $10^{-3}$, the average number of bits is around 140 bits compared to a maximum of 384 bits, with a variation mainly from 100 to 180 bits. With a target BER of $10^{-2}$, the variation increases and ranges from 130 to 250 bits.

For these systems, the number of outages is negligible since the maximum eigenvalue rarely falls into the outage region. For example, in a $(2,2)$ system, where the maximum eigenvalue tends to be the smallest for a MIMO system, the probability of outage is 0.0156 . This is even less for larger systems. Hence, distributional results are not of interest. However, for systems using all eigenchannels, the worst channel is that corresponding to the smallest eigenvalue, and outage is definitely an issue here. In Fig. 3, we see that, in a 64-subcarrier channel, a $(4,4)$ MIMO system uses less than 20 subcarriers more than half of the time and rarely uses more than 30 . For a $(2,2)$ system, the situation is better, and a variation from 10-60 useable channels is observed.

In our second set of simulations, we examine the LCR and the AFB for MIMO channel gains. First, for SISO OFDM systems, some selected results for the LCR and the AFB are plotted in Figs. 4 and 5. In Fig. 5, it can be seen that the simulated AFB saturates above a certain threshold level $T$. This is because the simulation results are generated from OFDM blocks with a finite bandwidth, and the largest fade bandwidth that we can

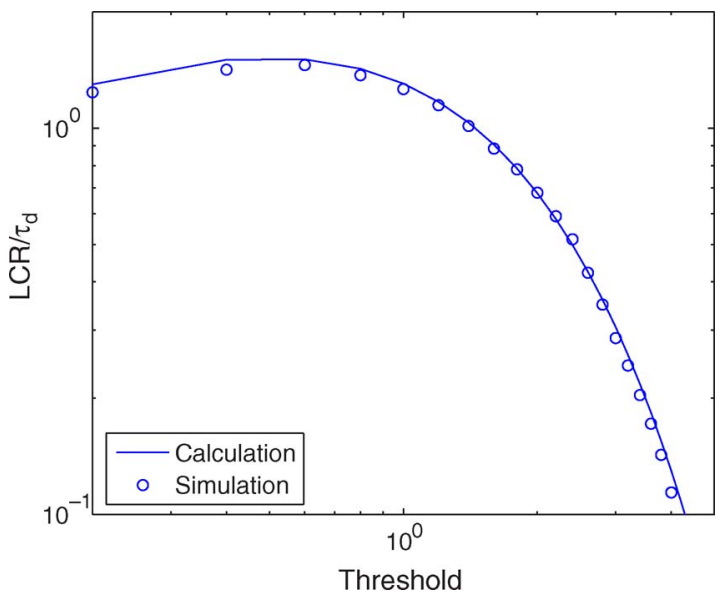

Fig. 4. Comparison between simulated and calculated normalized frequency LCRs for a SISO OFDM system $\left(N=64, \tau_{d}=100 \mathrm{~ns}\right)$.

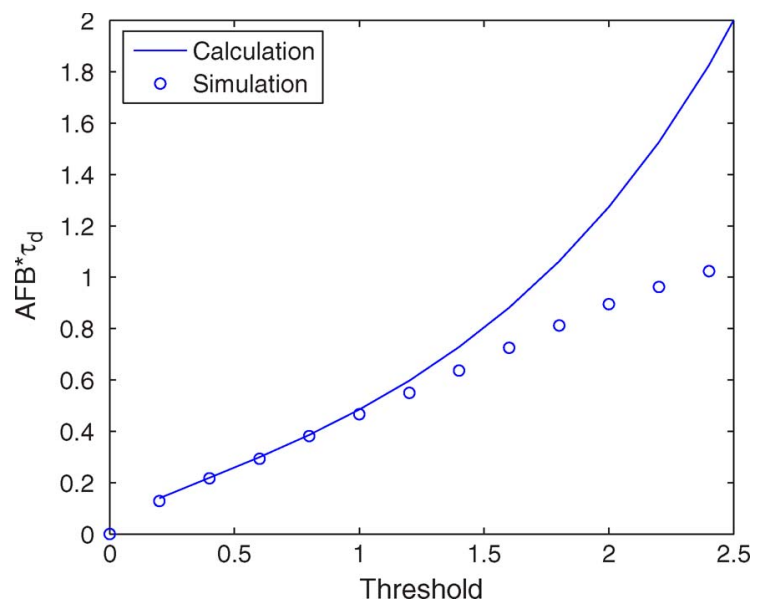

Fig. 5. Comparison between simulated and calculated AFBs for a SISO OFDM system $\left(N=64, \tau_{d}=100 \mathrm{~ns}\right)$.

possibly observe in the simulation is, therefore, constrained to $N \Delta f$. In contrast, the analysis yields the AFB for a continuous frequency process over an unbounded range. Hence, we might expect good agreement for fades of a small bandwidth, which might occur inside an OFDM block. For large bandwidth fades, the simulation will fall below the analysis, as these may be truncated by the finite bandwidth of the block. Fortunately, we are most interested in AFB values such that the channel gain is in a fade. These results are where the analysis is extremely accurate, as discussed later in detail.

As mentioned previously, when $\tau_{d}$ is sufficiently large, the channel response in the frequency domain becomes more discrete, and the LCR should be calculated using the joint probability method. The calculated LCR for a system with $\tau_{d}=250 \mathrm{~ns}$ is compared with our simulation results in Fig. 6 . The calculations using initial formula (37) are for the larger rms delay spread. The discrete version is clearly more accurate. Note that the continuous LCR is higher than the discrete version since, in continuous frequency, there can be level crossings between the discrete points, resulting in a higher value.

Turning now to MIMO OFDM systems, the LCR formula for the eigenmode in the frequency domain has been derived in (44). The accuracy of this formula for MIMO systems with 


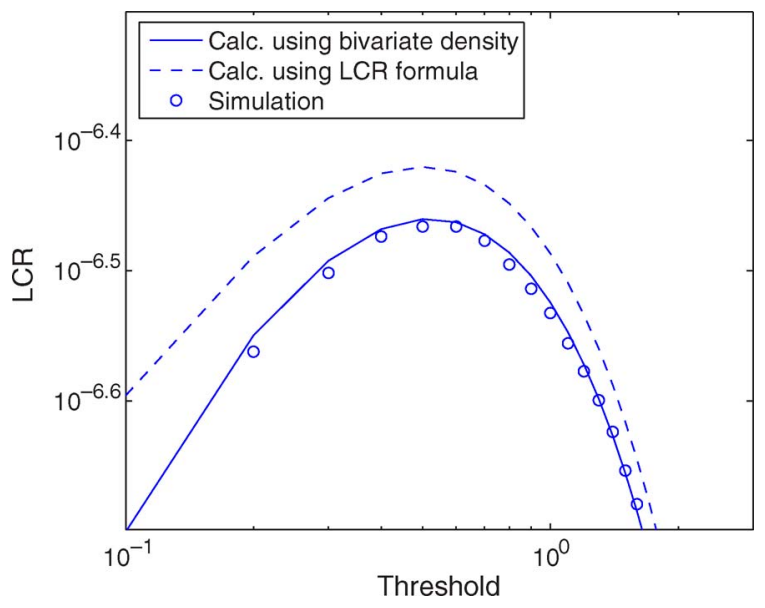

Fig. 6. Comparison between simulation and alternative formulas for the LCR of a SISO OFDM channel with lower correlation $\left(N=64, \tau_{d}=250 \mathrm{~ns}\right)$.

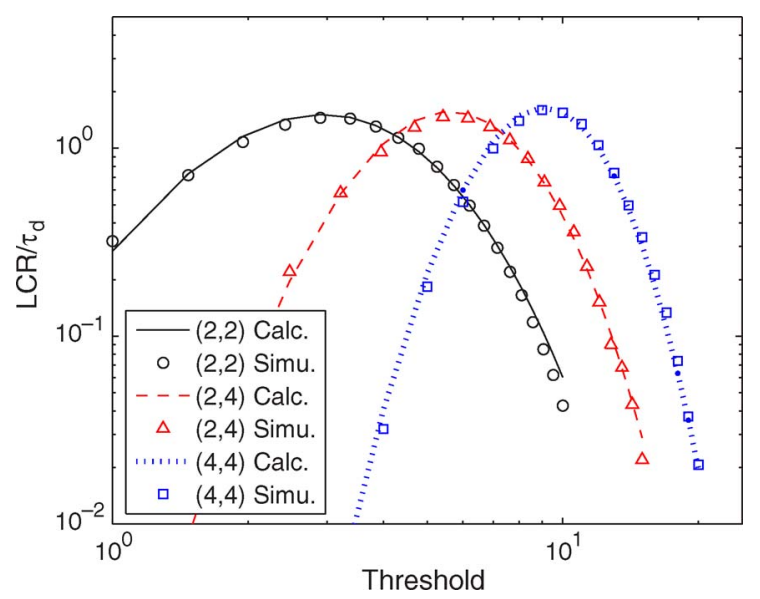

Fig. 7. Comparison between simulated and calculated normalized LCRs for the largest eigenmode in MIMO OFDM systems with different sizes $(N=64$, $\left.\tau_{d}=100 \mathrm{~ns}\right)$.

different sizes is exhibited in Fig. 7. Note that, although we are particularly interested in the largest eigenvalue in this paper, our formula is valid for any eigenvalues of interest. To show this, we have plotted $\mathrm{LCR}_{f}$ for the maximum and minimum eigenvalues of a $(3,3)$ system in Fig. 8. The good match between the simulation and the calculation for both eigenvalues is shown clearly. It is noticeable that the peak LCR for the smaller eigenvalue is much higher than that for the larger eigenvalue. This is plausible as the larger eigenmodes have substantially less frequency selectivity than the smaller ones [40].

We plot the AFB for the largest eigenvalue in a MIMO OFDM system with different $N_{R} \times N_{T}$ in Fig. 9. Once again, the simulated AFB saturates above a certain threshold level due to the limited bandwidth of the OFDM system. In addition, we note that the simulated AFB saturates at higher threshold values in systems with larger $N_{R} \times N_{T}$. Again, we see that, for all moderate to low thresholds, the analytical AFB is very accurate.

A close look at Fig. 9 yields considerable information. The threshold axes can be interpreted more clearly by computing the mean values for $\lambda_{\max }$, which are 3.5, 6.19, and 9.77 for $(2,2),(2,4)$, and $(4,4)$ systems, respectively. Hence, in all three figures, we see that the analytical AFB agrees with the simulations for all fades below thresholds up to the mean value

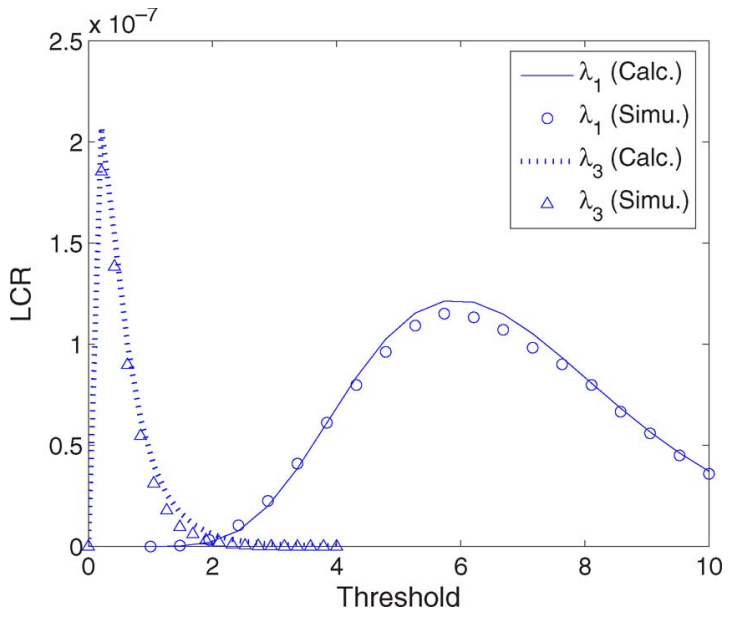

Fig. 8. Comparison between simulated and calculated normalized LCRs for largest and smallest eigenmodes in a $(3,3)$ MIMO OFDM system $(N=64$, $\left.\tau_{d}=100 \mathrm{~ns}\right)$.

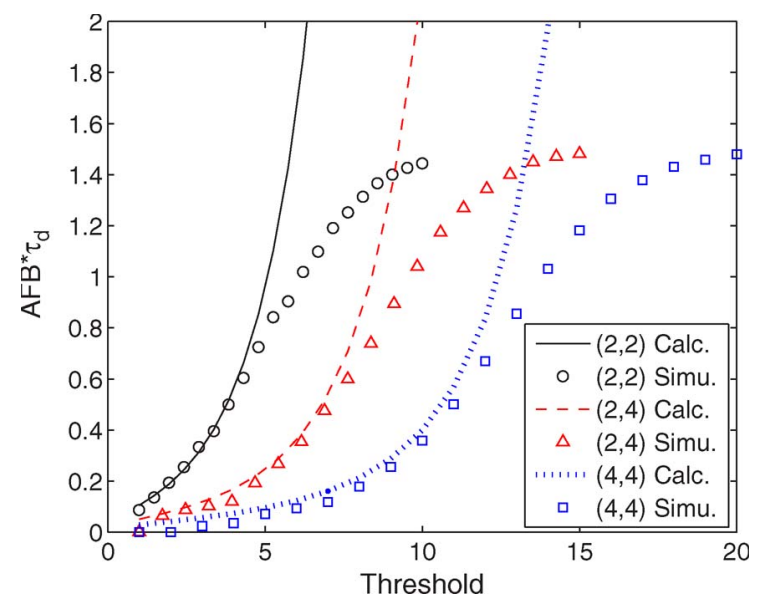

Fig. 9. Comparison between simulated and calculated AFBs for the largest eigenmode of a $(2,2)$ MIMO OFDM system $\left(N=64, \tau_{d}=100 \mathrm{~ns}\right)$.

of $\lambda_{\max }$. Thus, the analysis works extremely well in the region of interest: fades below thresholds that are small compared with $E\left[\lambda_{\max }\right]$.

Similarly, the $\mathrm{AFB} * \tau_{d}$ axes are best interpreted by converting the values from hertz to the equivalent number of subcarrier bins. In Fig. 9, $\mathrm{AFB} * \tau_{d}=1$ corresponds to 32 bins, and in Fig. 10, $\mathrm{AFB} * \tau_{d}=15$ corresponds to 480 bins. Hence, from Fig. 9, we see that the AFB below the mean of $\lambda_{\max }$ is approximately 16 bins. Fig. 10 shows that the analysis works well up to fades of average length around 300 bins. For deep fades, consider thresholds that are less than half the mean of $\lambda_{\max }$. In Fig. 9, such fades last, on average, between zero and six bins.

\section{CONCLUSION}

In this paper, we have considered some fundamental issues concerning the performance of adaptive MIMO OFDM systems and the behavior of the channel across frequency. Focusing on wideband channel variations in the frequency domain, we have considered both outage and data rate metrics and derived exact results for their means and variances. Furthermore, a CLT 


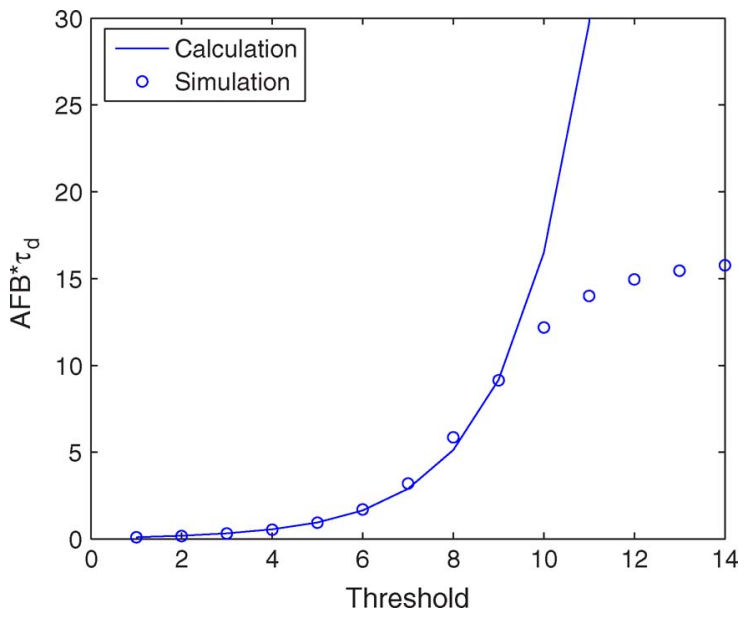

Fig. 10. Comparison between simulated and calculated AFBs for the largest eigenmode of a $(2,2)$ MIMO OFDM system $\left(N=512, \tau_{d}=100 \mathrm{~ns}\right)$.

has been developed, and the resulting Gaussian approximation has shown excellent agreement with our simulated results. Our results demonstrate that, although outage is a serious issue for the weaker eigenchannels, it very rarely occurs in MRTMRC systems. In terms of the data rate, we have observed wide variations of around $\pm 25 \%$ from the mean value. We have also derived very accurate approximations to the LCRs and the AFB of the MIMO eigenmodes. Our approximations can be further used to predict the time-varying characteristics of MIMO systems and their associated channel metrics so that an appropriate feedback/adaptation rate can be chosen in a judicious manner. The approximations are excellent for all thresholds that are less than the mean eigenmode and, therefore, yield accurate results for deep fades.

\section{APPENDIX}

Let $\left\{\boldsymbol{X}_{k}\right\}_{k=1}^{\infty}$ be a stationary mean-zero sequence of Gaussian vectors in $\mathbb{R}^{d}$, where $d=N_{R} \times N_{T}$. Set $\boldsymbol{X}_{k}=$ $\operatorname{vec}\left(\boldsymbol{H}_{k}\right)=\left(X_{k, 1}, X_{k, 2} \ldots, X_{k, d}\right)$, and let $g\left(\boldsymbol{X}_{k}\right)$ be a function on $\mathbb{R}^{d}$ with Hermite rank $\varphi(g)$ such that

$$
g\left(\boldsymbol{X}_{k}\right)=w_{i}, \quad \text { if } \lambda_{\max }^{(k)} \in R_{i}
$$

where $R_{i}=\left[T_{i}, T_{i+1}\right)$. With these definitions, we have $g\left(\boldsymbol{X}_{k}\right)=W_{k}$ as required. Now, the Hermite rank of $g(\cdot)$ is defined as follows for an arbitrary polynomial $P\left(\boldsymbol{X}_{k}\right)=$ $P\left(X_{k, 1}, X_{k, 2}, \ldots, X_{k, d}\right)$ and $\varphi(g) \triangleq \inf \varphi(g): \exists P\left(\mathbf{X}_{k}\right)$ of degree $\varphi(g)$, with

$$
E\left[\left\{g\left(\boldsymbol{X}_{k}\right)-E\left[g\left(\boldsymbol{X}_{k}\right)\right]\right\} P\left(\boldsymbol{X}_{k}\right)\right] \neq 0 .
$$

To produce our desired conclusion, we note that the Hermite rank $\varphi(g)$ is always nonnegative, and we demonstrate that it is at least 2 by showing that it is neither zero nor unity. Consider first a zero-order polynomial $P_{0}\left(\boldsymbol{X}_{k}\right)=\alpha_{0}$. Then, we can show that

$$
\begin{aligned}
E\left[\left\{g\left(\boldsymbol{X}_{k}\right)-E\left[g\left(\boldsymbol{X}_{k}\right)\right]\right\} P_{0}\left(\boldsymbol{X}_{k}\right)\right] & =\alpha_{0} E\left[g\left(\boldsymbol{X}_{k}\right)\right] \\
-\alpha_{0} E\left[g\left(\boldsymbol{X}_{k}\right)\right] & =0
\end{aligned}
$$

for all $\alpha_{0}$, and thus, $\varphi(g) \neq 0$. Next, consider a first-order polynomial

$$
P_{1}\left(\boldsymbol{X}_{k}\right)=\alpha_{0}+\sum_{i=1}^{d} \alpha_{i} X_{k, i} .
$$

To show that $\varphi(g) \neq 1$ and, hence, prove the desired result that $\varphi(g) \geq 2$, it suffices to show that $E\left[\left\{g\left(\boldsymbol{X}_{k}\right)-\right.\right.$ $\left.\left.E\left[g\left(\boldsymbol{X}_{k}\right)\right]\right\} P_{1}\left(\boldsymbol{X}_{k}\right)\right]=0 \quad$ for $\quad P_{1}\left(\boldsymbol{X}_{k}\right)=X_{k, i}$ with $i=$ $1, \ldots, d$. Furthermore, since $E\left[X_{k, i}\right]=0$ for all $i$, we only require $E\left[X_{k, i} g\left(\boldsymbol{X}_{k}\right)\right]=0$. We define $\boldsymbol{X}_{k}=\left(X_{k, i}, \boldsymbol{X}^{\prime}\right)$, where $\boldsymbol{X}^{\prime}$ contains the elements of $\boldsymbol{X}_{k}$ with $X_{k, i}$ removed. With this notation, we have

$$
\begin{aligned}
E & {\left[X_{k, i} g\left(X_{k, i}, \boldsymbol{X}^{\prime}\right)\right] } \\
& =\int_{-\infty}^{\infty} \int_{-\infty}^{\infty} \cdots \int_{-\infty}^{\infty} x g\left(x, \boldsymbol{x}^{\prime}\right) p\left(x, \boldsymbol{x}^{\prime}\right) d x d \boldsymbol{x}^{\prime} \\
& =\int_{-\infty}^{\infty} \int_{-\infty}^{\infty} \cdots \int_{-\infty}^{0} x g\left(x, \boldsymbol{x}^{\prime}\right) p\left(x, \boldsymbol{x}^{\prime}\right) d x d \boldsymbol{x}^{\prime} \\
& +\int_{-\infty}^{\infty} \int_{-\infty}^{\infty} \cdots \int_{0}^{\infty} x g\left(x, \boldsymbol{x}^{\prime}\right) p\left(x, \boldsymbol{x}^{\prime}\right) d x d \boldsymbol{x}^{\prime}
\end{aligned}
$$

where $p\left(x, \boldsymbol{x}^{\prime}\right)$ is the joint pdf of $X_{k, i}$ and $\boldsymbol{X}^{\prime}$. Substituting $u=-x$ and $\boldsymbol{u}^{\prime}=-\boldsymbol{x}^{\prime}$, after rearranging the terms, we find

$$
\begin{aligned}
E[ & \left.X_{k, i} g\left(X_{k, i}, \boldsymbol{X}^{\prime}\right)\right] \\
& =\int_{-\infty}^{\infty} \int_{-\infty}^{\infty} \ldots \int_{-\infty}^{0} x g\left(x, \boldsymbol{x}^{\prime}\right) p\left(x, \boldsymbol{x}^{\prime}\right) d x d \boldsymbol{x}^{\prime} \\
& +(-1)^{2 d+1} \int_{-\infty}^{\infty} \int_{-\infty}^{\infty} \ldots \int_{-\infty}^{0} u g\left(u, \boldsymbol{u}^{\prime}\right) p\left(u, \boldsymbol{u}^{\prime}\right) d u d \boldsymbol{u}^{\prime} \\
& =0
\end{aligned}
$$

where we have used the property that, in Rayleigh fading, the density $p\left(x, \boldsymbol{x}^{\prime}\right)$ is an even function, as is $g\left(x, \boldsymbol{x}^{\prime}\right)$.

The joint density function of $\boldsymbol{\lambda}$ and $\boldsymbol{w}$ is given in (24) and can be rewritten as

$$
\begin{aligned}
& f_{o}(\boldsymbol{w}, \boldsymbol{\lambda})=K e^{-\sum_{i=1}^{m} \delta w_{i}} e^{-\sum_{i=1}^{m} \delta \lambda_{i}}|V(\boldsymbol{\lambda})||V(\boldsymbol{w})| \\
& \times\left|\left(\lambda_{i} w_{j}\right)^{\frac{n-m}{2}} I_{n-m}\left(2 \sqrt{\mu \lambda_{i} w_{j}}\right)\right|
\end{aligned}
$$

where $K=\left(1-\rho^{2}\right)^{-m} \rho^{-m(n-1)} C_{o}, \quad \delta=\left(1-\rho^{2}\right)^{-1}$, and $V(\cdot)$ represents the Vandemonde matrix with $V(\boldsymbol{\lambda})=\left(\lambda_{j}^{i-1}\right)$ and determinant $|V(\boldsymbol{\lambda})|=\prod_{i<j}\left(\lambda_{i}-\lambda_{j}\right)$. Here, we have used the notation $\left(M_{i j}\right)$, which denotes an $m \times m$ matrix $\boldsymbol{M}$ with $(i, j)$ th element $M_{i j}$. We now apply a result of Chiani et al. [6, Corol. 2], which states that

$$
\int_{S}|\Phi(\boldsymbol{x})||\Psi(\boldsymbol{x})| \prod_{k=1}^{m} \xi\left(x_{k}\right) d \boldsymbol{x}=\left|\int_{a}^{b} \Phi_{i}(x) \Psi_{j}(x) \xi(x) d x\right|
$$

where $\boldsymbol{x}=\left(x_{1}, x_{2}, \ldots, x_{m}\right), \int_{S}$ represents the $m$-dimensional integration over the region $b \geq x_{1} \geq \cdots \geq x_{m} \geq a$, and 


$$
\operatorname{Prob}\left(\lambda_{1} \leq d, w_{1} \leq b\right)=K\left|\sum_{k=0}^{\infty} \frac{\mu^{k+\frac{n-m}{2}} \gamma(n-m+j+k, \delta b) \gamma(n-m+j+k, \delta d)}{\delta^{n-m+j+k} \delta^{n-m+i+k} k !(k+n-m) !}\right|
$$

$\Phi(\boldsymbol{x})$ and $\Psi(\boldsymbol{x})$ are $m \times m$ matrices with $(i, j)$ th elements of the form $\Phi_{i}\left(x_{j}\right)$ and $\Psi_{i}\left(x_{j}\right)$, respectively. We apply this result with $\boldsymbol{x}=\boldsymbol{w}, \Phi(\boldsymbol{w})=V(\boldsymbol{w}), \Psi(\boldsymbol{w})=$ $\left(\left[\lambda_{i} w_{j}\right]^{n-m / 2} I_{n-m}\left[2 \sqrt{\mu \lambda_{i} w_{j}}\right]\right)$, and $\xi\left(w_{i}\right)=e^{-\delta w_{i}}$. The result is given by

$$
\begin{aligned}
\int_{S} f_{o}(\boldsymbol{w}, \boldsymbol{\lambda}) d \boldsymbol{w}=K e^{-\sum_{i=1}^{m} \delta \lambda_{i}}|V(\boldsymbol{\lambda})| \\
\times\left|\int_{a}^{b} w^{i-1}\left(\lambda_{j} w\right)^{\frac{n-m}{2}} I_{n-m}\left(2 \sqrt{\mu \lambda_{j} w}\right) e^{-\delta w} d w\right| .
\end{aligned}
$$

Now, we apply (53) again, with $\boldsymbol{x}=\boldsymbol{\lambda}, \Phi(\boldsymbol{\lambda})=V(\boldsymbol{\lambda})$

$$
\Psi(\boldsymbol{\lambda})=\left(\int_{a}^{b} w^{i-1}\left(\lambda_{j} w\right)^{\frac{n-m}{2}} I_{n-m}\left(2 \sqrt{\mu \lambda_{j} w}\right) e^{-\delta w} d w\right)
$$

and $\xi\left(\lambda_{i}\right)=e^{-\delta \lambda_{i}}$. Also, the $m$-dimensional integral, denoted by $\int_{S}$, is replaced by $\int_{T}$, where $\int_{T}$ denotes the integration over the region $d \geq \lambda_{1} \cdots \geq \lambda_{m} \geq c$. This gives

$$
\begin{array}{rl}
\int_{T} \int_{S} f_{o}(\boldsymbol{w}, \boldsymbol{\lambda}) d \boldsymbol{w} & d \boldsymbol{\lambda}=K \mid \int_{c}^{d} \lambda^{i-1} \int_{a}^{b} w^{j-1}(\lambda w)^{\frac{n-m}{2}} \\
\times I_{n-m}(2 \sqrt{\mu \lambda w}) e^{-\delta w} d w e^{-\delta \lambda} d \lambda \mid .
\end{array}
$$

To compute (55), we require integrals of the form

$$
\begin{array}{r}
\int_{c}^{d}\left[\int_{a}^{b} w^{\frac{n-m}{2}+j-1} e^{-\delta w} I_{n-m}(2 \sqrt{\mu \lambda w}) d w\right] \\
\times \lambda^{\frac{n-m}{2}}+i-1 e^{-\delta \lambda} d \lambda .
\end{array}
$$

Using the series expansion for the modified Bessel function

$$
I_{n}(x)=\sum_{k=0}^{\infty} \frac{\left(\frac{x^{2}}{4}\right)^{k+\frac{n}{2}}}{k !(k+n) !}
$$

(56) can be rewritten as

$$
\begin{aligned}
\sum_{k=0}^{\infty} \frac{\mu^{k+\frac{n-m}{2}}}{k !(k+n-m) !} \\
\quad \times \int_{a}^{b} w^{\frac{n-m}{2}+j-1} e^{-\delta w} w^{\frac{n-m}{2}+k} d w \\
\quad \times \int_{c}^{d} \lambda^{\frac{n-m}{2}+i-1} e^{-\delta \lambda} \lambda^{\frac{n-m}{2}+k} d \lambda
\end{aligned}
$$

$$
\begin{aligned}
= & \sum_{k=0}^{\infty} \frac{\mu^{k+\frac{n-m}{2}}}{k !(k+n-m) !} \\
& \times\left[\int_{a}^{b} w^{n-m+j+k-1} e^{-\delta w} d w\right] \\
& \times\left[\int_{c}^{d} \lambda^{n-m+i+k-1} e^{-\delta \lambda} d \lambda\right] .
\end{aligned}
$$

Setting $a=c=0$ in (55) and using (58) result in (59), shown at the top of the page, where the integrals in (56) are expressed in terms of incomplete gamma functions. This gives the desired result in (26).

\section{REFERENCES}

[1] S. Catreux, V. Erceg, D. Gesbert, and R. W. Heath, "Adaptive modulation and MIMO coding for broadband wireless data networks," IEEE Trans. Commun., vol. 40, no. 6, pp. 108-115, Jun. 2002.

[2] J. C. Roh and B. D. Rao, "Adaptive modulation for multiple antenna channels," in Proc. 36th Asilomar Conf. Signals, Syst., Comput., Pacific Grove, CA, Nov. 3-6, 2002, vol. 1, pp. 526-530.

[3] D. Gesbert, M. Shafi, D.-S. Shiu, P. Smith, and A. Naguib, "From theory to practice: An overview of MIMO space-time coded wireless systems," IEEE J. Sel. Areas Commun., vol. 21, no. 3, pp. 281-302, Apr. 2003.

[4] S. M. Alamouti, "A simple transmit diversity technique for wireless communications," IEEE J. Sel. Areas Commun., vol. 16, no. 8, pp. 1451-1458, Oct. 1998.

[5] P. A. Dighe, R. K. Mallik, and S. S. Jamuar, "Analysis of transmit-receive diversity in Rayleigh fading," IEEE Trans. Commun., vol. 51, no. 4, pp. 694-703, Apr. 2003.

[6] A. Zanella, M. Chiani, and M. Z. Win, "Performance of MIMO MRC in correlated Rayleigh fading environments," in Proc. IEEE Veh. Technol. Conf., Stockholm, Sweden, May 30-Jun. 1, 2005, vol. 3, pp. 1633-1637.

[7] A. Maaref and S. Aissa, "Closed-form expressions for the outage and ergodic Shannon capacity of MIMO MRC systems," IEEE Trans. Commun., vol. 53, no. 7, pp. 1092-1095, Jul. 2005.

[8] M. Kang and M. S. Alouini, "Largest eigenvalue of complex Wishart matrices and performance analysis of MIMO MRC systems," IEEE J. Sel. Areas Commun., vol. 21, no. 3, pp. 418-426, Apr. 2003.

[9] K. K. Mukkavilli, A. Sabharwal, E. Erkip, and B. Aazhang, "On beamforming with finite rate feedback in multiple-antenna systems," IEEE Trans. Inf. Theory, vol. 49, no. 10, pp. 2562-2579, Oct. 2003.

[10] H. Bolcskei, D. Gesbert, and A. J. Paulraj, "On the capacity of OFDMbased spatial multiplexing systems," IEEE Trans. Commun., vol. 50, no. 2, pp. 225-234, Feb. 2002.

[11] C. C. Tan and N. C. Beaulieu, "Infinite series representations of the bivariate Rayleigh and Nakagami-m distributions," IEEE Trans. Commun., vol. 45, no. 10, pp. 1159-1161, Oct. 1997.

[12] M. Hagedorn, P. J. Smith, P. J. Bones, R. P. Millane, and D. Pairman, "A trivariate chi-squared distribution derived from the complex Wishart distribution," J. Multivariate Anal., vol. 97, no. 3, pp. 655-674, Mar. 2006.

[13] M. R. McKay, A. J. Grant, and I. B. Collings, "Largest eigenvalue statistics of double-correlated complex Wishart matrices and MIMO-MRC," in Proc. IEEE Int. Conf. Acoust., Speech, Signal Process., Toulouse, France, May 15-19, 2006, pp. IV-1-IV-4.

[14] A. Clark, P. Smith, and D. Taylor, "Instantaneous capacity of OFDM on Rayleigh-fading channels," IEEE Trans. Inf. Theory, vol. 53, no. 1, pp. 355-361, Jan. 2007. 
[15] L. J. Cimini, "Analysis and simulation of a digital mobile channel using orthogonal frequency division multiplexing," IEEE Trans. Commun., vol. COM-33, no. 7, pp. 665-675, Jul. 1985.

[16] A. F. Molisch, Ed., Wideband Wireless Digital Communications. Upper Saddle River, NJ: Prentice-Hall, 2001.

[17] G. L. Stuber, J. R. Barry, S. W. McLaughlin, Y. Li, M. A. Ingram, and T. G. Pratt, "Broadband MIMO-OFDM wireless communications," Proc. IEEE, vol. 92, no. 2, pp. 271-294, Feb. 2004.

[18] R. F. H. Fisher and J. B. Huber, "A new loading algorithm for discrete multitone transmission," in Proc. GLOBECOM, Nov. 18-22, 1996, pp. 724-728.

[19] A. J. Goldensmith and S. G. Chua, "Variable-rate variable-power MQAM for fading channels," IEEE Trans. Commun., vol. 45, no. 10, pp. 12181230, Oct. 1997

[20] W. C. Jakes, Ed., Microwave Mobile Communications. New York: IEEE Press, 1994.

[21] C. G. Khatri, "Distribution of the largest or the smallest characteristic root under null hypothesis concerning complex multivariate normal populations," Ann. Math. Stat., vol. 35, no. 4, pp. 1807-1810, Dec. 1964.

[22] J. C. Roh and B. D. Rao, "An efficient feedback method for MIMO systems with slowly time-varying channels," in Proc. IEEE Wireless Commun. Netw. Conf., Atlanta, GA, Mar. 21-25, 2004, pp. 760-764.

[23] D. Dardari, "Ordered subcarrier selection algorithm for OFDM-based high-speed WLANs," IEEE Trans. Wireless Commun., vol. 3, no. 5, pp. 1452-1458, Sep. 2004.

[24] S. Sampei and H. Harada, "System design issues and performance evaluations for adaptive modulation in new wireless access systems," Proc. IEEE, vol. 95, no. 12 , pp. 2456-2471, Dec. 2007.

[25] S. T. Chung and A. J. Goldsmith, "Degrees of freedom in adaptive modulation: A unified view," IEEE Trans. Commun., vol. 49, no. 9, pp. 15611571, Sep. 2001.

[26] M. Kang and M. S. Alouini, "Quadratic forms in complex Gaussian matrices and performance analysis of MIMO systems with cochannel interference," IEEE Trans. Wireless Commun., vol. 3, no. 2, pp. 418-431, Mar. 2004

[27] P. Doukhan, G. Oppenheim, and M. S. Taqqu, Eds., Theory and Applications of Long-Range Dependence. Boston, MA: Birkhauser, 2003.

[28] M. A. Arcones, "Limit theorems for nonlinear functionals of a stationary Gaussian sequence of vectors," Stat. Probab. Lett., vol. 22, no. 4, pp. 2242-2274, Oct. 1994.

[29] M. V. S. de Naranjo, "A central limit theorem for non-linear functionals of stationary Gaussian vector processes," Stat. Probab. Lett., vol. 22, no. 3 , pp. 223-230, Feb. 15, 1995.

[30] P. J. Smith and L. M. Garth, "Distribution and characteristic functions for correlated complex Wishart matrices," J. Multivariate Anal., vol. 98, no. 4, pp. 661-677, Apr. 2007.

[31] M. Chiani, M. Z. Win, and A. Zanella, "On the capacity of spatially correlated MIMO Rayleigh-fading channels," IEEE Trans. Inf. Theory, vol. 49, no. 10, pp. 2363-2371, Oct. 2003.

[32] P.-H. Kuo, P. J. Smith, and L. M. Garth, "Joint density for eigenvalues of two correlated complex Wishart matrices: Characterization of MIMO systems," IEEE Trans. Wireless Commun., vol. 6, no. 11, pp. 3902-3906, Nov. 2007.

[33] R. A. Silverman, "The fluctuation rate of the chi process," IRE Trans. Inf. Theory, vol. IT-4, no. 1, pp. 30-34, Mar. 1958.

[34] K. Witrisal, "OFDM air-interface design for multimedia communications," Ph.D. dissertation, Delft Univ. Technol., Delft, The Netherlands, 2002.

[35] P.-H. Kuo, P. J. Smith, L. M. Garth, and N. O'Connell, "Level crossing analysis for MIMO eigenmodes and associated channel metrics," IEEE Trans. Inf. Theory, Mar. 2007. to be published.

[36] P.-H. Kuo, "Channel variations in MIMO wireless communication systems: Eigen-structure perspectives," Ph.D. Dissertation, Univ. Canterbury, Christchurch, New Zealand, 2007.

[37] A. Zanella, M. Chiani, and M. Z. Win, "On the marginal eigenvalues distribution of Wishart matrices," Univ. Bologna, Bologna, Italy, Tech. Rep. IEIIT B0-07-06, Dec. 2006.

[38] R. Grunheid, E. Bolinth, and H. Rohling, "A blockwise loading algorithm for the adaptive modulation technique in OFDM system," in Proc. IEEE Veh. Technol. Conf., Atlantic City, NJ, Oct. 7-11, 2001, pp. 948-951.

[39] M. Lei and P. Zhang, "Subband bit and power loading for adaptive OFDM," in Proc. IEEE Veh. Technol. Conf., Orlando, FL, Oct. 6-9, 2003, pp. 1482-1486.

[40] S. Nanda, R. Walton, J. Ketchum, M. Wallace, and S. Howard, "A high-performance MIMO OFDM wireless LAN," IEEE Commun. Mag., vol. 43, no. 2, pp. 101-109, Feb. 2005.

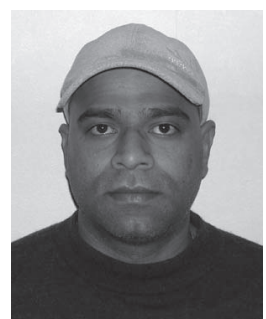

Krishna P. Kongara was born in Andhra Pradesh, India, in 1974. He received the B.Eng. degree in electronics and telecommunications and the M.Eng. degree in digital systems from the Institution of Electronics and Telecommunication Engineers, New Delhi, India, and Osmania University, Hyderabad, India, in 1997 and 1999, respectively. $\mathrm{He}$ is currently working toward the Ph.D. degree with the University of Canterbury, Christchurch, New Zealand.

From 1999 to 2001, he was a Digital Signal Processing Design Engineer with Hellosoft Pvt. Ltd., Hyderabad. From 2001 to 2003, he was with the 3G R\&D Laboratories, NEC Australia, Melbourne, Australia. From 2003 to 2006, he was with Tait Electronics Ltd., Christchurch. His research interests include multicarrier transmission, high-speed data over narrow-band PMR channels, MIMO-OFDM, adaptive modulation, simulcast transmission, software-defined radio, and real-time implementation of communication systems.

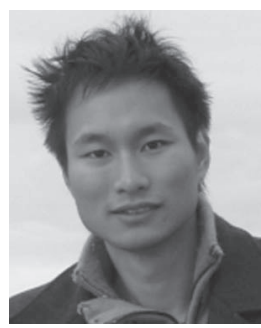

Ping-Heng Kuo was born in Pingtung, Taiwan. He received the B.S. (with Honors) and Ph.D. degrees in electrical and computer engineering from the University of Canterbury, Christchurch, New Zealand, in 2003 and 2007, respectively.

He was a Research Intern with the Communication and Networking Laboratory, Samsung Advanced Institute of Technology, Suwon, Korea. He is currently a Signal Processing Engineer with the Taiwan Design Center, Atheros Communications Inc., Hsinchu, Taiwan. He is the author of the book Fluctuations of MIMO Wireless Channels: Analysis Based on Brownian Motion Theory (VDM Verlag, 2008). His research interests include statistical characterization of mobile channels, MIMO-OFDM wireless techniques, scheduling algorithms for multiuser wireless communication systems, cooperative and relay networks, and Global Positioning System receiver architectures.

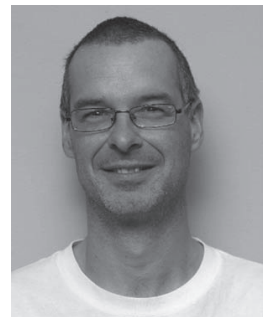

Peter J. Smith (M'93) received the B.Sc. degree in mathematics and the $\mathrm{Ph} . \mathrm{D}$. degree in statistics from the University of London, London, U.K., in 1983 and 1988, respectively.

From 1983 to 1986, he was with the Telecommunications Laboratories, GEC Hirst Research Centre. From 1988 to 2001, he was a Lecturer in statistics with Victoria University, Wellington, New Zealand. Since 2001, he has been a Senior Lecturer and an Associate Professor of electrical and computer engineering with the University of Canterbury, Christchurch, New Zealand. His research interests include the statistical aspects of design, modeling, and analysis for communication systems, particularly antenna arrays, MIMO, cognitive radio, and relays.

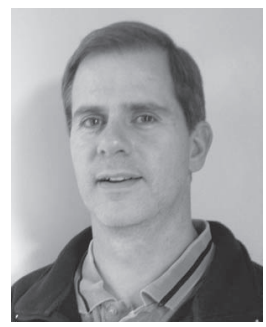

Lee M. Garth received the B.S.E. degree (magna cum laude) from Princeton University, Princeton, NJ, in 1987 and the M.S. and Ph.D. degrees from the University of Illinois at Urbana-Champaign in 1989 and 1996, respectively.

He had summer employment with Raytheon Company, GTE Corporation, and MITRE Corporation. From 1990 to 1996, he was a Senior Engineer with the Techno-Sciences, Inc., Urbana. From 2000 to February 2008, he was a faculty member with the Department of Electrical and Electronic Engineering, University of Canterbury, Christchurch, New Zealand. In 2006, he held a visiting appointment with the Samsung Advanced Institute of Technology, Suwon, Korea. Since March 2008, he has been a Senior Principal Engineer with the Advanced Systems and Technologies Division, BAE Systems, Merrimack, $\mathrm{NH}$. His research interests include signal detection, array processing, adaptive equalization, and statistical signal processing with applications to communications systems.

Dr. Garth is a member of Tau Beta Pi. From 1996 to 2000, he was a member of the Advanced Data Communications Group of Bell Laboratories within Lucent Technologies, Holmdel, NJ.

Alan Clark, photograph and biography not available at the time of publication. 